CASE REPORT

\title{
Scrub Typhus Infection: A Rare Pneumonia Mimic
}

\author{
Kaustubh Mohite ${ }^{1}$, Anilkumar Sapare ${ }^{2}$, Hiremath Sagar ${ }^{3}$
}

\begin{abstract}
Scrub typhus is a common infection in tropical countries affecting mainly the pediatric population. It is caused by Orientia tsutsugamushi with characteristic pathological change being focal or disseminated vasculitis. Main clinical symptoms involve fever, rash, headache, myalgia along with edema of the face and limbs. Lobar pneumonia is not a common manifestation of scrub typhus infection. Here, we discuss a case of a girl who presented with unilateral neck swelling with lobar pneumonia who turned up to be positive for scrub typhus infection.
\end{abstract}

Keywords: Pediatric, Pneumonia, Scrub typhus.

Pediatric Infectious Disease (2022): 10.5005/jp-journals-10081-1314

\section{INTRODUCTION}

Scrub typhus is a common pediatric infection caused by Orientia tsutsugamushi. The characteristic pathological change involves focal or disseminated vasculitis due to the destruction of endothelial cells and perivascular infiltration of leukocytes. Regional and generalized lymphadenitis is common. Pulmonary complications involve interstitial edema, interstitial pneumonia, and hemorrhage caused by vasculitis. Rarely, children may present with lobar pneumonia. A low threshold for diagnosis with familiarity with radiological findings aids early initiation of treatment and thereby reduces mortality. Here, we present a child with lobar pneumonia who on detailed examination and workup turned out to be positive for scrub typhus infection.

\section{Case Description}

An 11-year-old female child was brought to the hospital with chief complaints of persistent high-grade fever for 7 days. This was associated with a progressively increasing swelling over the right side of the neck which was non-tender, non-mobile with no signs of active inflammation (Fig. 1A). She was given some oral medications in other hospitals but the fever persisted. Fine needle aspiration cytology of the lymph node was suggestive of reactive lymphadenitis. On admission to our hospital, clinical examination revealed cervical lymphadenopathy. Lymph nodes measured $3 \times$ $3 \mathrm{~cm}$ in the right posterior triangle with palpable adjacent lymph nodes of smaller size. Chest examination revealed impaired notes on percussion and diminished breath sounds in right lower lung zones. There was no lymph node enlargement seen elsewhere, no hepatosplenomegaly. Detailed examination failed to reveal eschar over the body surface. Ultrasonography of the neck revealed enlarged multiple bilateral cervical lymph nodes with thickened cortex. Abdominal ultrasonography showed splenomegaly $(12 \mathrm{~cm})$. A complete blood count documented showed leukocytopenia (total WBC count-4,000/ $\mu \mathrm{L})$. X-ray chest showed bilateral diffuse haziness with predominantly left lower zone opacity and obliteration of left costophrenic angle suggestive of left-sided pleural effusion (Fig. 1B). To do a detailed evaluation of the changes observed in chest X-ray, a CT scan was done which was suggestive of left pleural effusion with basal atelectasis and patchy consolidation of the left lower lobe (Figs $1 \mathrm{C}$ to E). ESR documented was $40 \mathrm{~mm} /$ hour and CRP levels were $88.94 \mathrm{mg} / \mathrm{L}$ which indicated an active infection. Blood

\author{
${ }^{1-3}$ Department of Pediatrics, Narayana Health City, Bengaluru, \\ Karnataka, India
}

Corresponding Author: Anilkumar Sapare, Department of Pediatrics, Narayana Health City, Bengaluru, Karnataka, India, Phone: +91 9880410427, e-mail: aksapare@hotmail.com

How to cite this article: Mohite K, Sapare A, Sagar H. Scrub Typhus Infection: A Rare Pneumonia Mimic. Pediatr Inf Dis 2022;4(1):19-20.

Source of support: Nil

Conflict of interest: None

culture was sent, and she was started on intravenous ceftriaxone (100 mg/kg/day). Blood culture was reported to be sterile. With a clinical suspicion of pulmonary tuberculosis, a Mantoux test was done, bronchoalveolar lavage and pleural tap were collected for Gene X-pert, AFB stain, and AFB culture. All the above reports were negative but fever persisted. The mother gave a history of travel to her village 10 days before the onset of fever. Hence, scrub typhus IgM by ELISA was done which was positive (cut-off value $>0.5$ ). She was started on intravenous doxycycline (10 mg/kg) after which her fever dramatically reduced and she became afebrile in the next 48 hours. Neck swelling gradually subsided and she was discharged on oral doxycycline for a total of 7 days (Fig. 1F). Since the ELISA report was positive for scrub typhus and the child showed a good response to IV doxycycline, the diagnosis of scrub typhus pneumonia was established.

\section{Discussion}

Scrub typhus is seen commonly in most parts of the world but is difficult to diagnose. If undiagnosed and untreated in the pediatric age group, the fatality rate can be as high as $30-35 \%$, but when diagnosed properly they are often easily treated. ${ }^{1}$ Children present with non-specific signs and symptoms. In temperate countries like India, an outbreak of scrub typhus is observed mainly from October through January when mites are active. Fever is the most common presentation followed by headache and myalgia as observed by various studies in India. ${ }^{2}$ The painless chigger bite occurs mainly over the axilla or genital region, which are often missed during an examination. An eschar is seen in $50 \%$ at the bite site in primary infection. It begins as a papule, which progressively enlarges, undergoes central necrosis, and later 

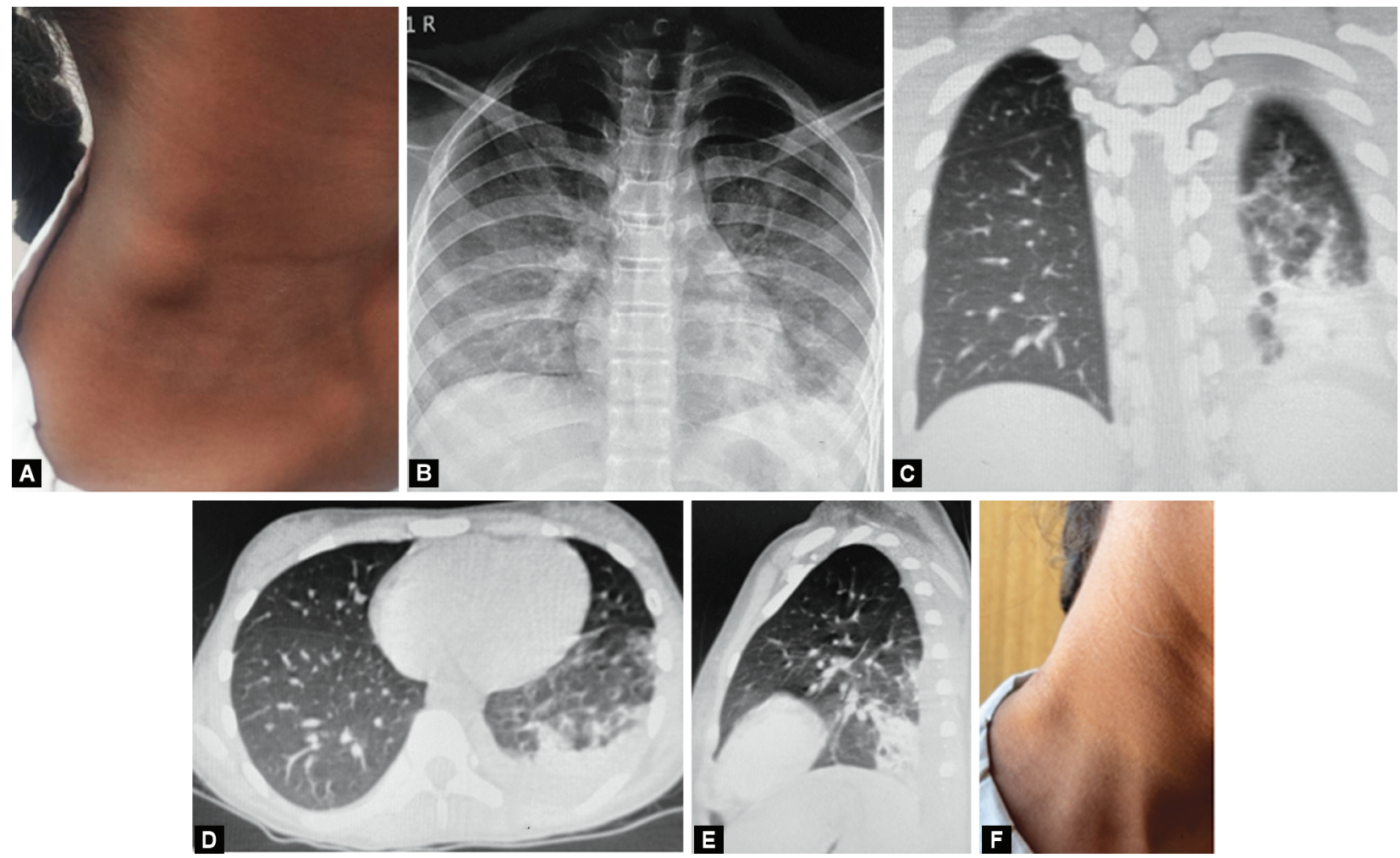

Figs 1A to F: Case of scrub typhus: (A) Neck swelling at presentation; (B) Chest X-ray PA view showing left lower zone opacity with obliteration of left costophrenic angle suggestive of left-sided pleural effusion; (C) Coronal section of CECT chest showing lobar pneumonia of left lower lobe; (D) Axial view of CECT chest showing involvement of posterior zone of left lower lobe; (E) Sagittal view showing involvement of the same left lung region; (F) Decreased size of the neck swelling after 48 hours of treatment with doxycycline

forms a blackened crust to resemble a lesion of a cigarette burn. ${ }^{3}$ Although the rash is considered as a hallmark of this infection, it is neither seen at presentation nor in all patients which makes the diagnosis difficult. ${ }^{4}$

Rickettsia is known to characteristically cause vascular injury in the visceral organs, most severely affecting the lungs, leading to pneumonia, diffuse alveolar damage, and increased vascular permeability. ${ }^{5}$ Classical manifestations include recurrent fever, headache, and myalgia. Examination reveals hepatosplenomegaly, peripheral edema. A significant number of children may show rash varying from maculopapular to non-blanching vasculitic rash. In severe forms, they can have encephalitis, seizures, myocarditis, severe hepatic dysfunction, and rarely acute kidney injury. The predominant mechanism of organ injury is vasculitis. In most parts of our country where investigation facilities are sparse, empirical use of doxycycline in clinically suspected children leads to rapid resolution of pyrexia in 24-48 hours. This rapid deffervescence of fever in children with persistent pyrexia can be used to diagnose rickettsial illness. Diagnosis of scrub typhus by ELISA method has proven to be an effective and economical way as this test has good sensitivity and specificity. ${ }^{6}$

The consideration of scrub typhus is especially important in tropical countries where the prevalence of this disease is higher. A combination of antimicrobials like penicillin, cephalosporins, and aminoglycosides are used as first-line therapy in the treatment of community-acquired pneumonia. However, not all patients respond to the given line of therapy. In such scenarios, it is important to consider the possibility of scrub typhus, especially if other systemic signs become more apparent, namely generalized edema, hepatosplenomegaly, and hypertransaminesemia. A very meticulous search for eschar in axillae, inguinal areas, scalp, and hairlines will yield the diagnosis. The rapid clinical response to doxycycline and resolution of pneumonia will be seen in children with scrub typhus.

\section{ACKnOWledgments}

We would like to express our gratitude toward the patients and their parents for being a part of this study.

\section{References}

1. Batra HV. Spotted fevers \& typhus fever in Tamil Nadu. Indian J Med Res 2007;126(2):101.

2. Kamarasu K, Malathi M, Rajagopal V, et al. Serological evidence for wide distribution of spotted fevers \& typhus fever in Tamil Nadu. Indian J Med Res 2007;126(2):128.

3. Mahajan SK, Mahajan SK. Neuropsychiatric manifestations of scrub typhus. J Neurosci Rural Pract 2017;8(3):421. DOI: 10.4103/jnrp. jnrp_44_17.

4. Mahajan SK, Rolain JM, Sankhyan N, et al. Pediatric scrub typhus in Indian Himalayas. Indian J Pediat 2008;75(9):947-949. DOI: 10.1007/ s12098-008-0198-z.

5. Walker DH, Parks FM, Betz TG, et al. Histopathology and immunohistologic demonstration of the distribution of rickettsia typhi in fatal murine typhus. Am J Clin Pathol 1989;91(6):720-724. DOI: 10.1093/ajcp/91.6.720.

6. Varghese GM, Rajagopal VM, Trowbridge P, et al. Kinetics of $\operatorname{Ig} M$ and $\lg G$ antibodies after scrub typhus infection and the clinical implications. Int J Infect Dis 2018;71:53-55. DOI: 10.1016/j. ijid.2018.03.018. 